\title{
Factors That Influence Human Error and Patient Safety in the Pre-hospital Emergency Care Setting: Perspectives of Emergency Care Practitioners in a Middle-income Country
}

\section{Mugsien Rowland}

Nelson Mandela University, None

Anthonio Oladele Adefuye ( $\sim$ AdefuyeAO@ufs.ac.za)

University of the Free State Faculty of Health Sciences https://orcid.org/0000-0003-2380-1487

Original research

Keywords: emergency care practitioners, human error, patient safety, pre-hospital emergency care

Posted Date: September 3rd, 2021

DOI: https://doi.org/10.21203/rs.3.rs-850377/v1

License: (c) (1) This work is licensed under a Creative Commons Attribution 4.0 International License. Read Full License 


\section{Abstract}

Background: Delivering pre-hospital emergency care has the potential to be hazardous, and the patient could experience an adverse event. Despite these potential, yet known, threats, little is known about patient safety in the pre-hospital care setting, in contrast to in-hospital care. In South Africa, there are no reports on patient safety and human error issues in the pre-hospital care setting. This study investigated the perspectives of emergency care practitioners (ECPs) in South Africa on the types of errors that occur in the pre-hospital emergency care setting, as well as factors that influence patient safety and precipitate errors during pre-hospital care.

Methods: This research was designed as an exploratory study that used a questionnaire administered on 2000 ECPs to obtain their perceptions on factors the influence human error and patient safety in the prehospital emergency care environment.

Results: Of the 2000 questionnaires distributed, 1,510 were returned, giving a response rate of $76 \%$. Analysis of the respondents' responses in relation to the types of human errors in the pre-hospital environment yielded five dominant themes, namely errors relating to poor judgement, poor skill/knowledge, fatigue, and communication, and human error. According to the participants, inadequate equipment, environmental factors, personal safety concerns, practitioner's incompetence, and ineffective teamwork are the top five factors that influence patient safety in the pre-hospital emergency care setting. The majority $(65.1 \% ; p<0.001)$ of public sector ECPs reported that they had not received training on patient safety, nor do they have a protocol for managing medical error at their workplace $(65.7 \%$; $p<$ 0.007).

Conclusion: In conclusion, this study investigated paramedics' views on types of errors that occur in the pre-hospital emergency care setting, and factors that influence patient safety and precipitate errors during pre-hospital care. It was found that public-sector emergency medical service (EMS) in South Africa seldom train their staff on patient safety or have a protocol for managing medical error. The study advocates that, to overcome medical errors, EMS in South Africa should establish a culture of safety that focuses on system improvement and personnel training.

\section{Background}

The healthcare professional, being human, has been described as a small, but probably the most errorprone part, of a modern healthcare system that is dependent on an array of non-human systems.(1) There is a growing realization that human error adversely influences patient safety and contributes significantly to morbidity and mortality in a modern healthcare system.(2) Preserving patient safety in contemporary medical practice is a global problem; it has been estimated that $10 \%$ of patients admitted to a hospital are exposed to harmful incidents, and $2 \%$ die because of medical error (attributed to human error).(3-5) While these estimates might be true for developed countries, there is currently very limited literature on the impact of error on patient outcome in developing countries.(2) Estimates indicate that the 
medical error rate in developing countries (including South Africa) is $8.2 \%$ of hospital admissions.(6) Most reports and investigations into patient safety and human error in the healthcare setting are hospitalbased, and there is a global shortage of investigations into patient safety and human error in the prehospital emergency care setting.(7)

Pre-hospital emergency medical care can be provided anywhere, including at the roadside, in a home, or in a public setting, with associated threats to safety that are unspecified, unpredictable, and/or uncontrollable.(8) In this milieu, the ECP must think, move, and react swiftly to reduce threats to life and limb, and to stabilise the patient quickly.(8) Furthermore, delays, interruptions, or distractions are common in this high-risk environment, and may contribute to errors in judgment, medication choices and delivery, or in executing procedures.(8) ECPs in South Africa are not exempted from these errors. Despite these potential, yet known, threats, there have been no reports on patient safety and human error issues in the pre-hospital care setting in South Africa. In a Canadian study, errors due to clinical reasoning and decision-making as the most important factors that influence patient safety in a pre-hospital emergency care setting.(9) This study investigated the perspectives of ECPs in South Africa on the types of errors that occur in the pre-hospital emergency care setting, as well as factors that influence patient safety and precipitate errors during pre-hospital care. Identifying these errors and factors that precipitate them, will assist emergency care providers in developing strategies to mitigate them.

\section{Methods}

This research was designed as an exploratory study that utilised a survey tool to obtain both quantitative and qualitative data.

\section{Study population}

The study population comprised ECPs in South Africa (employed in either the state or private sector) who were, at the time of the study, registered with the Health Professions Council of South Africa (HPCSA). The full list and the contact details (e-mail addresses) of registered ECPs were obtained, with permission, from the HPCSA. At the time of this study, the total number of registered ECPs was $N=24,970$ (sample frame). The estimated minimum sample size was calculated as $n=1,000$ (at $99 \%$ confidence level (CL) and margin of error (MOE) 0.04). Simple random sampling technique was utilised and the target population comprised $n=2,000$ ECPs. The study sample consists of respondents who consented to complete the questionnaire and thereby participate in the study.

\section{Questionnaire survey}

Questionnaire items were compiled from factors identified by prior studies, $(10,11)$ following a thorough literature review and content analysis. The literature review included medical subject headings search terms, such as medical error, human error in pre-hospital care, medical error in pre-hospital care, patient safety, and patient safety in pre-hospital care. The databases used to access articles were Google Scholar, MEDLINE, PubMed, CINAHL, SABINET, Science Direct, and Directory of Open Access Journals. 
Concepts were identified to formulate both open-ended and closed-ended questions; the closed-ended questions were answered using adapted Likert-scale ranking. The open-ended questions allowed for the investigation of perceptions and provided contextual depth to the data collected. The questionnaire consisted of five sections. The first section, Section A, focused on participants' demographic information (i.e. data on participants' age, gender, registration cadre, employment profile and province of employment). Section B, an open-ended section, was designed to obtain information about participants' perspectives on the different types of human error in the pre-hospital care setting. Section $C$ focused on identifying factors participants perceived as contributing to human error in the pre-hospital care setting. In Section D, the statement, "List some of the factors influencing patient safety in the pre-hospital care setting", was used to obtain participants' views on factors that may influence patient safety in the prehospital care setting. Section E obtained information on aspects of organizational culture and attitudes towards patient safety and human error in the pre-hospital care setting using a five-point Likert scale with the options "Never", "Once in a while", "About half the time", "Most of the time" and "Always". For the purpose of analysis, these options were reduced to three-point Likert scale, as follows: "Never" + "Once in a while" = "Never"; "Most of the time" + "Always" = Always; and "About half the time". The questionnaire was administered electronically via the QuestionPro survey management system. A cover letter that elucidated the objectives of the study, as well as the conditions for study participation, was sent by e-mail to all potential participants. By clicking on the link to the questionnaire, participants automatically agreed tacitly to participate in the study. A waiting period of one week from date of delivery was given for participants to complete the survey, after which repeated electronic reminders to complete the questionnaire - one per week - were sent for three consecutive weeks. The survey was conducted from 26 January 2021 to 26 February 2021.

\section{Pilot study}

A pilot study was executed prior to the official start of data collection, to test the suitability of the study's design and methods, the chosen data collection method, and the overall structure of the questionnaire. The pilot study involved 10 ECPs. An e-mail that contained an information sheet and a request to participate in the pilot study, as well as an electronic links to the questionnaire, were sent to each participant. The findings of the pilot study confirmed the adequacy of the chosen data collection method and the overall structure of the questionnaire. The participants in the pilot study did not recommend changes to the structured questionnaire. They estimated the total time needed to complete the questionnaire to be approximately 30 minutes.

\section{Validity}

Questionnaire validity was achieved by comparing the questionnaire elements with those of similar studies and by conducting a pilot study. In addition, a faculty evaluation committee subjected the questionnaire to review and approval.(12)

\section{Reliability}


The closed-ended questions in the questionnaire were grouped into subsets and analysed for reliability by using Cronbach's alpha.(13-15) Cronbach's analysis for the subset of questions in the questionnaire ranged from 0.57 to 0.78 , thus, suggesting that the items within the subsets were related to one another and would provide reliable answers to the questions they were designed to investigate.

\section{Data analysis}

Statistical analysis of all numerical data was performed by using the statistical software package TIBCO Statistica ${ }^{\text {TM }}$ version 13.5.0.17. Results are presented in contingency tables as frequencies and percentages. Chi-squared test and Cramér's $V$ were used to examine the existence and the strength of an association between cross-tabulated variables. Spearman's rho correlation was used to analyse the strength of association between factors that influence human error, and the perceived types of human error. Responses to open-ended questions were analysed qualitatively using Atlas.ti 8.3 software (Scientific Software Development, Germany). Iterative inductive coding was done with the assistance of a co-coder, and attention was paid to emerging patterns and themes.

\section{Results}

\section{Participant Demographics}

Out of 2,000 questionnaires distributed, 1510 were returned, giving a response rate of $76 \%$. Of the participants, $62.6 \%(n=909)$ were men and $37.4 \%(n=542)$ were women (ratio 2:1). The median age was 37.4 years $( \pm S D=1.14$ years $)$, with the majority $(40.3 \% ; n=592)$ within the age bracket $25-34$ years and having worked for an average of 11.2 years $( \pm S D=8.4)$. Participants registration cadres are as follows: Basic Ambulance Assistant (BAA) (38.6\%; $n=566)$; Ambulance Emergency Assistant (AEA) $(29.4 \% ; n=$ 432); Emergency Care Practitioner (ECP) (9.4\%; $n=138)$; Emergency Care Technician (ECT) $(9.1 \% ; n=$ 134); Critical Care Assistant (CCA) $(8.4 \% ; n=124)$; Emergency Care Assistant (ECA) $(0.2 \% ; n=3)$; and Other $(4.8 \% ; \mathrm{n}=71)$.

Regarding the nature of their employment, the majority $(83.4 \% ; n=1,186)$ of the participants reported that they worked as operational emergency care practitioners, $11.3 \%(n=160)$ worked as emergency care support staff, and only $5.3 \%(n=5)$ worked in EMS communications. The majority $(53.1 \% ; n=736)$ of the participants were employed in the private sector, while $46.9 \%(n=649)$ worked in the public sector. Eighthundred-and-ninety-six (62.9\%) participants indicated that their EMS base is located in an urban area, $20.1 \%(n=286)$ indicated that they work in rural areas, and $17.1 \%(n=243)$ worked in semi-rural areas.

\section{Participants' perspectives on types of human error in the pre-hospital emergency care setting}

Analysis of the participants' responses to the types of human error that occur in the pre-hospital environment yielded five dominant themes, namely poor judgement error, poor skill/knowledge-based error, fatigue-related error, human error, and communication error. 
Poor judgement error. The majority of the participants reported that poor judgement errors often occur as misdiagnoses, and incorrect medication or dosages, as reported by quotes \#1-\#8.

\#1 "Missed diagnosis"

\#2 "Incorrect interpretation of diagnosis"

\#3 "Wrong drug dosages"

\#4 "Misdiagnosis - mistreatment"

\#5 "Miscalculated dosages"

\#6 "Misdiagnosis, wrong dose/drug"

\#7 "Wrong drug calculations"

\#8 "Misjudgement of symptoms"

Poor skill/knowledge-based error. According to the participants, poor skill/knowledge-based error in the pre-hospital care setting can represent as poor clinical reasoning and poor patient management (cf. quotes \#9-\#11).

\#9 "Lack in clinical reasoning leading to wasted time on scene"

\#10 "Management related errors"

\#11 "incorrectly managing a patient due to lack of knowledge"

In addition, participants listed lack of proper training and failure to attend continuous professional development programmes as among the factors that can precipitate poor skill-based errors (cf. quotes \#12-\#15)

\#12 "Not doing refresher courses"

\#13 "Lack of proper training"

\#14 "lack of adequate training"

\#15 "Not doing clinical updates"

Fatigue-related error. Participants reported that fatigue-related errors often present as lapses in concentration, leading to mistakes when attending to patients (cf. Quotes \#15-\#17); fatigue is attributed to work overload, lack of rest, stress and burnout, as reported by quotes \#16-\#23.

\#16 "lapse in concentration i.e. mistakes" 
\#17 "Loss of concentration"

\#18 "Poor concentration"

\#19 "overloading of work"

\#20 "overwork/tired"

\#21 "No resting enough before shift"

\#22 "stress when dealing with difficult situations, panic"

\#23 “Burnout"

Human error. Participants indicated that bad attitudes and behaviours, lack of confidence, and fear leading to negligence in practice constitute some of the human errors occurring in the pre-hospital emergency care setting (cf. Quotes \#24-\#29).

\#24 "Lack of confidence"

\#25 "Fear"

\#26 "Bad attitudes"

\#27 "poor practitioner attitude"

\#28 "Negligence"

\#29 “Carelessness, Attitude"

Communication error. As described by the participants, communication errors in the pre-hospital emergency care setting could occur because of poor or miscommunication between ECPs, language barriers between the practitioners and patients, and failure to communicate with patients (cf. Quotes \#30-\#33)

\#30 "Lack of effective communication"

\#31 "Miscommunication”

\#32 "Communication barriers"

\#33 "not communicating with the patient"

Factors participants perceive as contributing to human error in the pre-hospital emergency care setting

Analysis of participants' responses regarding factors that are perceived to contribute to human error in the pre-hospital emergency care setting generated eight emerging themes, namely work related 
fatigue/stress, insufficient education and training, insufficient clinical knowledge and experience, unsafe work environment, poor communication skills, being overconfident, poor leadership and management practices, and intimidation by and pressure from senior colleagues.

Work related fatigue/stress. The majority of the participants reported that work-related fatigue and/or stress represent a major contributing factor to human error in the pre-hospital emergency care environment (cf. Quotes \#34-\#39). In addition, work related fatigue/stress was found to correlate with positively fatigue-related error $(r=0.24 ; p \leq 0.01)$.

\#35 "Fatigue"

\#36 "Stress at work"

\#37 "Exhausted/burnt out personnel"

\#38 "Stress induced freezing"

\#39 "Fatigue and practitioner burnout"

Insufficient education and training. Participants also indicated that insufficient training as well as not attending continuous development programmes could contribute to human error in the pre-hospital emergency care setting as shown in quotes \#40-\#44. A high positive correlation was found between insufficient education and training and poor skill/knowledge-based error $(r=0.92 ; p \leq 0.01)$.

\#40 "Poor training"

\#41 "Lack of knowledge and training"

\#42 "Lack of education/training/revision"

\#43 "Not enough training"

\#44 "Lack of continuous development"

Insufficient clinical knowledge and experience. Limited clinical knowledge, as well as lack of experience in pre-hospital emergency care, were also suggested by the participants as factors that may lead to human error when attending to patients in the pre-hospital setting (cf. Quotes \#45-\#51). This factor was found to correlate positively and significantly with poor skill/knowledge-based error $(r=0.83 ; p \leq 0.01)$.

\#45 "Lack of exposure or lack of experience"

\#46 "Not knowing the work"

\#47 "Lack of knowledge"

\#48 "Lack of clinical knowledge" 
\#49 "Insufficient skills set or knowledge"

\#50 "Lack of adequate experience"

\#51 "No knowledge"

Unsafe work environment. Participants of this study, furthermore, indicated that fear for personal safety and stress associated with working in an unsafe environment contribute to human error in the prehospital setting, as exemplified by quotes \#51-\#57.

\#51 "Unsafe environment"

\#52 "Danger... crime.. .gangs... rioting"

\#53 "Extreme stress due to threat to your life"

\#54 "Fear of being mugged end up doing mistakes if working in the red zone"

\#55 "Red zones. Safety"

\#56 "Environmental danger"

\#57 "Fear of personal safety"

Poor communication skills. Miscommunication, inability to communicate effectively, and lack of communication was cited by the participants as factors that can contribute to human error in the prehospital emergency care setting (cf. Quotes \#57-\#61). Similarly, the factor, poor communication skills correlates positively with communication error $(r=0.84 ; p \leq 0.01)$.

\#57 "Poor communication"

\#58 "Inability to communicate effectively"

\#59 "Lack of communication"

\#60 “Communication breakdown"

\#61 "Poor communication skills"

Overconfidence. A "know-it-all" attitude or feeling of being overconfident about one's clinical skills was also identified as one the factors that may contribute to errors in pre-hospital care, as shown by quotes \#62-\#64.

\#62 "Over confidence"

\#63 "Knowing it all" 
\#64 "Be over confident"

Poor leadership and management practices. Participants, furthermore, reported that a lack of good leadership and management practices at an emergency scene could contribute to errors during prehospital care, as highlighted by quotes \#65-\#68.

\#65 "Poor Leadership Practices"

\#66 "Poor Management Practice”

\#67 "Without clear leadership or direction"

\#68 "Collapse of the chain of command..."

Intimidation by and pressure from senior colleagues. Lastly, participants were of the opinion that intimidation by and pressure from senior colleagues can also precipitate human error during pre-hospital emergency care (cf. Quotes \#69-\#80).

\#69 "Pressure from Superiors to meet timelines"

\#70 "Fear of higher qualified practitioners"

\#80 "Intimidation by senior medics"

Factors perceived by participants as influencing patient safety in the pre-hospital emergency care setting

In this section of the questionnaire, participants were asked to list factors that, in their own opinion, influence patient safety in the pre-hospital emergency care setting. Analysis of participants' responses generated six dominant themes, as shown in Fig. 1. According to participants, inadequate equipment, environmental factors, personal safety concerns and practitioners' incompetence are the top four influencers of patient safety in the pre-hospital emergency care setting.

\section{Organizational culture and attitudes about patient safety and human error in the pre-hospital care setting Protocol for managing medical errors}

First, participants were asked to indicate whether their organization has a protocol for managing medical errors. Results as shown in Table 1 reveal a weak but significant association between the type of workplace and the presence of a protocol for managing medical error. The majority (65.7\%) of the respondents who work in the public sector indicated that they do not have a protocol for managing medical error at their workplace $\left(X^{2}=28.8\right)$; Cramer's $\left.V=0.2 ; p<0.001\right)$. 
Table 1

Availability of protocol for managing medical error at workplace

\begin{tabular}{|lll|}
\hline & \multicolumn{2}{c|}{ Protocol for managing medical error at the workplace } \\
\hline Type of workplace & Yes (\%) & No (\%) \\
\hline Public sector & 42.2 & 65.7 \\
Private sector & 57.8 & 34.3 \\
\hline
\end{tabular}

\section{Training to ensure patient safety during pre-hospital emergency care}

In this regard, participants were asked to indicate how often they received training on ensuring patient safety during pre-hospital emergency care. The results show that the majority of the participants working in either the private $(53.7 \%)$ or public $(65.1 \%)$ sector has ever received training on patient safety $\left(\chi^{2}=\right.$ 14.1); Cramer's V $=0.14 ; p<0.007)$ (Table 2).

Table 2

How often participants received training on patient safety

\begin{tabular}{|lll|}
\hline & \multicolumn{2}{l|}{ Type of workplace } \\
\hline Frequency of training on patient safety & Public (\%) & Private (\%) \\
\hline Never & 65.1 & 53.7 \\
\hline About half the time & 11.9 & 14.0 \\
\hline Always & 23.0 & 32.3 \\
\hline
\end{tabular}

\section{Discussion}

Findings of this study about the male to female ratio - 2:1 - are consistent with findings by other studies and confirms that the pre-hospital emergency medical care environment in South Africa is a maledominated field. $(16,17)$ This finding suggests that there may be a gender bias in the EMS profession. The median age of 37.4 years is consistent with findings by Mothibi, Jama and Adefuye (2019), and suggest that a relatively young population dominates the EMS profession in South Africa.(16) Historically, the EMS profession in South Africa owes its existence to short vocational qualification courses, such as the BAA, AEA, and CCA. An increase in the number of training colleges and institutions accredited by the HPCSA to offer short courses in the early 90 s increased the numbers of ECPs trained. Thus, by 2018, the majority of pre-hospital ECPs registered with the HPCSA were BAA and AEA practitioners.(18) This change is reflected in the findings of this study, which report that the majority (68\%) of participants were registered as BAA and AEA. In South Africa, more healthcare workers are employed in the private sector than in the public sector.(19) Similarly, the findings of this study report that the majority of ECP participants were employed in the private sector. Factors such as high patient loads, 
long working hours, inadequate resources and occupational hazards are some of the reasons cited by healthcare professionals for avoiding employment in the public health system.(20) It is, therefore, plausible that this might be the case for the ECPs who participated in this study too.

One of the dominant themes identified from the participants' responses with regard to the types of human errors that occur in the pre-hospital emergency care setting, is "poor judgement error", which was perceived as diagnostic and medication errors. Diagnostic errors were identified as causing one the major adverse events that patients experience in clinical practice.(21) While the actual diagnostic error rates in clinical practice are difficult to determine, it has been estimated that about $10-15 \%$ of all rendered diagnoses are incorrect.(22) In the pre-hospital emergency care setting, diagnostic errors are recognised as the most frequent cause of allegations of negligence, and contribute to a worldwide high mortality rate every year.(23) This suggests that diagnostic errors might be responsible for some of the mortality recorded in the pre-hospital setting in South Africa.(24) An accurate diagnosis by a paramedic is essential for directing immediate treatment and subsequent care, and can improve the patient's outcome.(25) Administering medications urgently during emergency care makes the process prone to error. Our finding, namely that administration of incorrect drug dosages is a type of human error that occurs in the prehospital setting, is consistent with findings of the literature.(26) Factors such as poor medication knowledge, wrong calculations, and nomenclature issues have been attributed to medication error.(27) It is, therefore, plausible that such factors may underlie the medication error as perceived by the participants of this study.

Bijani et al. (2021) report that good clinical knowledge, experience, and skills contribute to an ECP's professional capabilities, which are essential for making good clinical decisions and avoiding error.(28) This suggests that an opposing state, i.e., poor skills and knowledge, and limited experience, can contribute to error during pre-hospital care. To this end, participants listed "practitioner's incompetence" as factors that can influence patient safety in the pre-hospital setting. In addition, participants identified "Insufficient clinical knowledge and experience" and "Insufficient education and training" as factors that are perceived to contribute to error during pre-hospital emergency care. These factors were found to correlate positively with "Poor skill/knowledge-based error", and suggests that insufficient clinical knowledge, and too little education and experience precipitate poor skill/knowledge-based errors. This finding highlights the need for adequate and effective education and training.

Fatigue is a complex phenomenon that affects physical characteristics, cognition, behaviour and mental health of an individual.(29) Growing body of evidence suggests that fatigue can negatively affect a paramedic's performance. Increased levels of fatigue are associated with poor cognitive function,(30) impaired performance, and increased error and accident rates.(29) Similarly, participants of this study reported "work related fatigue/stress" as a factor that can contribute to perceived error in the pre-hospital emergency care setting. Work-related fatigue/stress correlates positively with "Fatigue-related error", a type of error identified by the participants. Moreover, participants listed fatigue and stress as factors that can influence patient safety in the pre-hospital setting. Fatigue management approaches, such as limiting shift length and promoting adequate rest between shifts, can be used to combat fatigue-related error. 
Poor communication is an important cause of adverse events in healthcare system, and can result in medical error.(31) Likewise, participants in our study reported that "poor communication skill" can contribute to errors, and this was found to correlate positively with "communication error" in the prehospital emergency care setting. It has been suggested that routine team checklist briefing can help to improve team communication.(31)

Our findings also reveal that participants identified "human error" as type of error in the pre-hospital care setting. As the participants indicated, human error was associated with negative behaviours and emotions. In their study that investigated causes, consequences, and emotional responses to medical errors, Bari, Khan and Rathmore (2016) report that lack of knowledge is often associated with negative emotions, such as fear and lack of confidence, and could lead to error (31) - again emphasizing the role of adequate education and training in averting human error in the pre-hospital care setting.

Overconfidence has been identified as a source of cognitive bias that may influence reasoning and medical decision-making, and can cause errors.(32) Similarly, participants of this study reported that being overconfident is a factor that can contribute to errors in the pre-hospital care setting.

While they are on duty, ECPs in South Africa often suffer physical abuse, are assaulted and subject to violent behaviours by patients, bystanders and criminals.(33) EMS personnel have been robbed, assaulted or shot at in the line of duty. The constant fear for their own life and personal safety experienced by these ECPs can lead to error and adverse events relating to patients. Hence, participants of this study reported that "unsafe work environment" and "personal safety concerns" could contribute to error and influence patient safety in the pre-hospital emergency care setting. Including hostile environment awareness training (HEAT) programmes in the undergraduate emergency medical care curriculum has been suggested as a strategy to enable ECPs cope with hostile environments.(34) HEAT programmes focus on developing an individual's ability to assess situations, recognise specific risk factors, and, where possible, avoiding these risks.(34) The role of health leadership should be centred on identifying priorities and providing strategic direction in order to improve health services.(35) To improve patient outcomes in the pre-hospital setting, ECPs must demonstrate competence regarding both effective leadership and clinical leadership skills.(36) To this end, ECPs who participated in this study recognised "poor leadership and management practices" as factors that could contribute to errors during pre-hospital emergency care. The hierarchical parameters entrenched by the level of qualification/training in the pre-hospital environment ensures that the followership role is often assigned to lower-level cadres, while practitioners with higher qualifications assume leadership roles.(37) This practice can lead to intimidation by and unsolicited pressure from colleagues, which can be a source of errors during prehospital emergency care, as indicated by the participants of this study.

Our finding that the participants of this study perceived inadequate equipment and environmental issues as to be factors that could influence patient safety in the pre-hospital emergency care setting, is consistent with similar findings by Guise, Meckler, O'Brien et al. (2015).(38) Participants highlighted how a shortage of proper tools/equipment to undertake job functions can influence patient's safety negatively. Environmental factors such as hazardous scenes, crowds, the weather, and crime are also some of the 
factors reported by the participants to influence patient safe. This is consistent with similar findings by Vincent-Lambert and Mottershaw (2018). (39)

Teamwork between pre-hospital emergency care providers and other healthcare professionals is essential for managing acute clinical emergencies. It has been reported that inadequate teamwork is often responsible for preventable medical errors. $(40,41)$ Similarly, participants of our study reported that "ineffective teamwork" could influence patient safety in the pre-hospital emergency care setting. It is recommended that deliberate teaching and training on principles and practice of crisis resource management is undertaken to enhance teamwork skills of paramedics.(37)

Medical error is a serious public health problem and a leading cause of death globally. It is not easy to find the consistent cause of errors and, even if the cause is found, providing a consistent viable solution that minimises the likelihood of recurrent errors is difficult.(42) Healthcare providers and institutions have been encouraged to implement protocols and processes to reduce medical error.(43) In this study, the majority of the participants who worked in the public sector indicated that they do not have a protocol for managing medical error at their workplace, nor have they ever received training on patient safety. We, therefore, advocate that public emergency care providers in South Africa establish a culture of safety that focuses on system improvement to overcome medical errors.

\section{Conclusion}

In conclusion, this study investigated paramedics' views on types of errors that occur in the pre-hospital emergency care setting, as well as factors that influence patient safety and precipitate errors during prehospital care. The study found that public-sector emergency medical service (EMS) in South Africa seldom train their staff on patient safety or have a protocol for managing medical error. Implementation of strategies that enhances education and training; clinical skill development; teamwork skills; fatigue management; and leadership skills can help prevent some the errors identified. In addition, simple practices, such as implementing viable safety protocols to prevent and manage medical error, and ensuring that ECPs are continuously trained on maintaining patient safety during pre-hospital care can also assist in this regard.

\section{Limitations}

A major limitation of this study was that participants did not answer all the questions in the questionnaire.

\section{Declarations}

\section{Ethical considerations}

Approval to conduct this study was obtained from the Health Sciences Research Ethics Committee of the Faculty of Health Sciences, University of the Free State (Ref. No. UFS-HSD2020/0462/2807). Permission 
was also obtained from the HPCSA.

\section{Availability of data and materials}

The datasets used and/or analysed during the current study are available from the corresponding author on reasonable request.

\section{Conflict of interest}

All authors declare that there is no conflict of interest.

\section{Funding}

Funding was obtained from the Health and Welfare Sector Education and Training Authority (HWSETA) of South Africa.

\section{Authors' contributions}

MR conceptualised the study, collected the data and drafted the manuscript; AA supervised the study, reviewed and analysed the data, and gave expert input in drafting the manuscript.

\section{Acknowledgements}

The authors will like to thank all ECPs who participated in this study.

\section{References}

1. Reason JT. The human contribution: unsafe acts, accidents and heroic recoveries. Farnham: Ashgate Publishing, Ltd.; 2008.

2. Bashir A, Kong V, Buitendag J, Manchev V, Bekker W, Bruce J, et al. An analysis of adverse events and human error associated with the imaging of patients at a major trauma centre in South Africa. SAMJ. 2019;109(9):693-7.

3. Runciman B, Walton M. Safety and ethics in healthcare: a guide to getting it right. Farnham: Ashgate Publishing, Ltd.; 2007.

4. Hagiwara MA, Nilsson L, Strömsöe A, Axelsson C, Kängström A, Herlitz J. Patient safety and patient assessment in pre-hospital care: a study protocol. Scand J Trauma, Resusc. 2016;24(1):1-7.

5. Reason J. Delivering Patient Safety: Facing the Facts. Edmonton, Canada: Canadian Patient Safety Institute. 2007.

6. Wilson RM, Michel P, Olsen S, Gibberd R, Vincent C, El-Assady R, et al. Patient safety in developing countries: retrospective estimation of scale and nature of harm to patients in hospital. BMJ. $2012 ; 344$. 
7. Sayre MR, White LJ, Brown LH, McHenry S. National EMS Research Agenda. Prehospital emergency care. 2002;6(3 Suppl):S1-43.

8. Patterson PD, Yealy DM. Safety in the Prehospital Emergency Medical Services Setting. Patient Saftey Network. 2019.

9. Bigham BL, Bull E, Morrison M, Burgess R, Maher J, Brooks SC, et al. Patient safety in emergency medical services: executive summary and recommendations from the Niagara Summit. CJEM. 2011;13(1):13-8.

10. Bigham B, Brooks S, Maher J. Patient safety in emergency medical services: advancing and aligning the culture of patient safety in EMS. . Edmonton, Alberta: Canadian Patient Safety Institute; 2011.

11. Hagiwara MA, Magnusson C, Herlitz J, Seffel E, Axelsson C, Munters M, et al. Adverse events in prehospital emergency care: a trigger tool study. BMC emergency medicine. 2019;19(1):1-10.

12. Ringsted C, Hodges B, Scherpbier A. 'The research compass': An introduction to research in medical education: AMEE Guide No. 56. Med Teach 2011;33(9):695-709.

13. Bland JM, Altman DG. Statistics notes: Cronbach's alpha. BMJ. 1997;314(7080):572.

14. DeVellis RF. Scale Development: Theory and Applications. Fourth ed. Thousand Oaks, Carlifornia, United States: SAGE Publications; 2016. 280 p.

15. Taber KS. The use of Cronbach's alpha when developing and reporting research instruments in science education. Res Sci Educ. 2018;48(6):1273-96.

16. Mothibi JD, Jama M, Adefuye AO. Assessing the knowledge of emergency medical care practitioners in the Free State, South Africa, on aspects of pre-hospital management of psychiatric emergencies. Pan Afr Med J. 2019;33.

17. Butler MW, Adefuye AO. Assessing the knowledge of emergency medical care personnel in the Free State, South Africa, on aspects of paediatric pre-hospital emergency care. Pan Afr Med J. 2019;32.

18. Sobuwa S, Christopher LD. Emergency care education in South Africa: past, present and future. Australas J Paramedicine. 2019;16.

19. Econex N. Note 4 NHI Note 4: Supply Constraints. Stellenbosch: Econex. 2009.

20. George G, Reardon C. Preparing for export? Medical and nursing student migration intentions postqualification in South Africa. Afr J Prim Health Care Fam Med 2013;5(1):1-9.

21. Neale G, Hogan H, Sevdalis N. Misdiagnosis: analysis based on case record review with proposals aimed to improve diagnostic processes. Clin Med. 2011;11(4):317.

22. Graber ML. The incidence of diagnostic error in medicine. BMJ Qual Saf. 2013;22(Suppl 2):ii21-ii7.

23. Pelaccia T, Messman AM, Kline JA. Misdiagnosis and failure to diagnose in emergency care: causes and empathy as a solution. Patient Educ Couns. 2020;103(8):1650-6.

24. Meel B. Pre-hospital and hospital traumatic deaths in the former homeland of Transkei, South Africa. $\mathrm{J}$ clin forensic med. 2004;11(1):6-11.

25. Koivulahti O, Tommila M, Haavisto E. The accuracy of preliminary diagnoses made by paramedics-a cross-sectional comparative study. Scand J Trauma, Resusc. 2020;28(1):1-7. 
26. Nguyen A. Bad medicine: preventing drug errors in the prehospital setting. JEMS. 2008;33(10):94100.

27. Lesar TS, Briceland L, Stein DS. Factors related to errors in medication prescribing. Jama. 1997;277(4):312-7.

28. Bijani M, Abedi S, Karimi S, Tehranineshat B. Major challenges and barriers in clinical decisionmaking as perceived by emergency medical services personnel: a qualitative content analysis. BMC emergency medicine. 2021;21(1):1-12.

29. Ramey S, MacQuarrie A, Cochrane A, McCann I, Johnston CW, Batt AM. Drowsy and dangerous? Fatigue in paramedics: an overview. IJP. 2019.

30. Harrison Y, Horne JA. The impact of sleep deprivation on decision making: a review. Journal of experimental psychology. 2000;6(3):236.

31. Bari A, Khan RA, Rathore AW. Medical errors; causes, consequences, emotional response and resulting behavioral change. Pak J Med Sci. 2016;32(3):523.

32. Berner ES, Graber ML. Overconfidence as a cause of diagnostic error in medicine. Am J Med. 2008;121(5):S2-S23.

33. Holgate R. The opinion of emergency medical service personnel regarding safety in pre-hospital emergency care practice. Johannesburg: University of the Witwatersrand. 2015.

34. CraigVincent-Lambert, Westwood R. Students' views on the need for hostile environment awareness training for South African emergency medical care students. Afr J Health Prof Educ. 2019;11(1):12-5.

35. Reich MR, Javadi D, Ghaffar A. Introduction to the special issue on "effective leadership for health systems". Health Syst reform. 2016.

36. Martins JCA, de Sousa ACV, Abrantes ARD, da Silva Pinto CS, de Almeida Gomes Cl, Martins DJO, et al. Communication and leadership in emergency situations: Systematic literature review and recommendations for practice. Clin Nurs Stud. 2018; 6 (2):55-62.

37. Rowland M, Adefuye AO, Vincent-Lambert C. The need for purposeful teaching, learning and assessment of crisis resource management principles and practices in the undergraduate prehospital emergency care curriculum: A narrative literature review. Australas J Paramedicine. 2021;18.

38. Guise J-M, Meckler G, O'Brien K, Curry M, Engle P, Dickinson C, et al. Patient safety perceptions in pediatric out-of-hospital emergency care: children's safety initiative. J Pediatr. 2015;167(5):1143-8. e1.

39. CraigVincent-Lambert, Mottershaw T. Views of emergency care providers about factors that extend on-scene time intervals. Afr J Emerg Med. 2018;8(1):1-5.

40. Hughes AM, Gregory ME, Joseph DL, Sonesh SC, Marlow SL, Lacerenza CN, et al. Saving lives: A meta-analysis of team training in healthcare. J Appl Psychol. 2016;101(9):1266.

41. Aron D, Headrick L. Educating physicians prepared to improve care and safety is no accident: it requires a systematic approach. BMJ Qual Saf. 2002;11(2):168-73. 
42. Rodziewicz TL, Houseman B, Hipskind JE. Medical Error Reduction and Prevention. Treasure Island, Florida: StatPearls Publishing; 2018.

43. La Pietra L, Calligaris L, Molendini L, Quattrin R, Brusaferro S. Medical errors and clinical risk management: state of the art. Acta Otorhinolaryngol Ital. 2005;25(6):339.

\section{Figures}
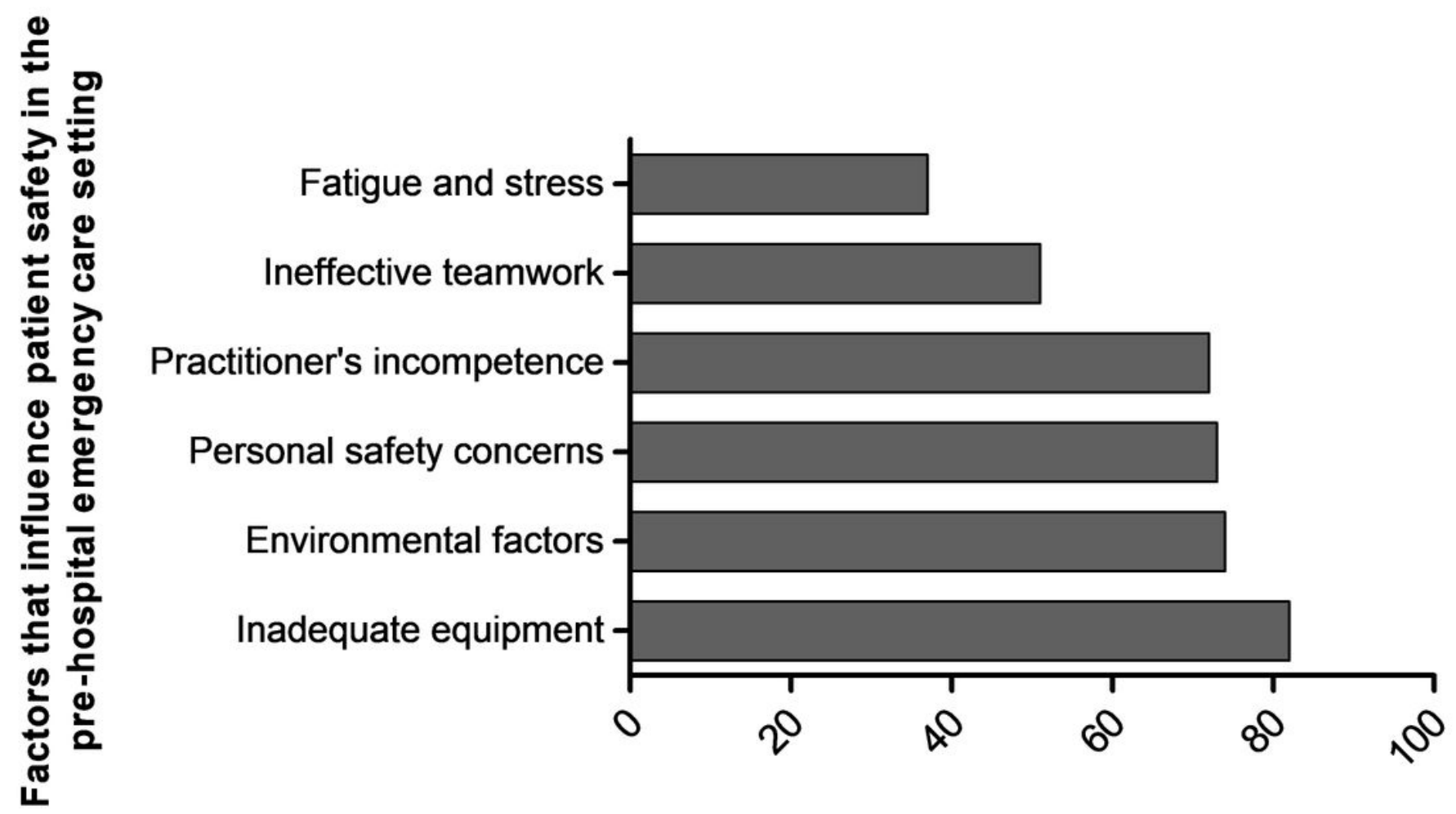

Frequency count

Figure 1

Participants' views on factors that influence patient safety in pre-hospital emergency care setting 